\title{
A Literature Review: Artificial Intelligence Impact on the Recruitment Process
}

\author{
J. FRAIJ ${ }^{1}$, V. LÁSZLÓ ${ }^{2}$ \\ ${ }_{1}$ University of Debrecen Faculty of Economics and Business, Department of Business Informatics, \\ jihadfraij@hormail.com \\ ${ }_{21}$ University of Debrecen Faculty of Economics and Business, Department of Business Informatics, \\ varallyai.laszlo@econ.unideb.hu
}

\begin{abstract}
This paper aims to review artificial intelligence (AI) implementation in the Human Resources Management (HRM) recruitment processes. A systematic review was adopted in which academic papers, magazine articles as well as high rated websites with related fields were checked. This study's findings should contribute to the general understanding of AI's impact on the HRM recruitment process. It was impossible to track and cover all topics related to the subject. However, the research methodology seems reasonable and acceptable as it covers a good number of articles related to the core subject area. The results and findings were almost precise that using AI is advantageous in the area of recruitment as technology can serve best in this area. Moreover, time, effort, and boring daily tasks are transformed into computerized, making adequate space for humans to focus on more important subjects related to boosting performance and development. Acquiring automation and cognitive insights as well as cognitive engagement in the recruitment process would make it possible for systems to work similarly to the human brain in terms of data analysis and the ability to build an effective systematic engagement to process the data in an unbiased, efficient and fast way.
\end{abstract}

Keywords: Artificial intelligence, recruitment process, Staffing, sourcing of candidates, candidate communication, human bias.

\section{Introduction}

The business world is witnessing rapid changes, with which human resources departments find themselves standing in front of a new reality. The prediction of the World Economic Forum (Future Jobs Report 2018) that seventy-five million current jobs will pass from sight by 2022, and one hundred thirty-three million new jobs will be established, thanks to robotics and artificial intelligence (AI) (Leopold, Ratcheva and Zahidi, 2018). It has always been the same conflict between humans and machines. Machines have already changed many jobs and replaced humans in so many tasks. However, the idea has changed nowadays to establish human-technology cooperation, boosting human abilities and capabilities. Nowadays, organizations are striving to find talented candidates with multi-skills qualifications to compete in the global market. This paper aims to offer a deeper understanding of AI's use in the recruitment process and AI's impact on three main processes: screening, human bias, and the best-fit candidate. 


\section{Methodology}

A systematic Literature review was adopted in this paper. It reviewed the previous studies on AI and the recruitment process. The researchers adopted a methodology based on \{Formatting Citation\}. The methodology process is based on six principles, as shown in Figure (1):

Mapping the

filed through a

scoping review
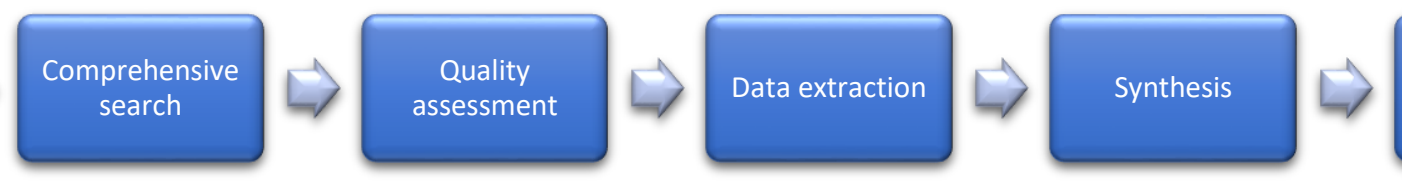

Write-up

Figure 1. Represents the six principles methodology of (Jesson, Matheson and Lacey, 2011)

Researchers managed the study as follows. At first, two research questions were developed based on the research interest. A question with two folds was prepared, firstly, which studies have focused on the impact of AI on the Recruitment process. Secondly, what are the results of those studies taking into consideration the exclusion and inclusion standards? Afterward, specific keywords were chosen to select suitable studies. The researchers have used ("Artificial Intelligence") AND ("Human Resources Management" OR "Recruitment" OR "Talent Acquisition"). The database that the researchers have used are Sage, Scopus, Springer link, and Emerald. Besides, grey literature like non-academic reports were also been studied. The results of the search were presenting a huge number of studies. The third step was important to minimize the numbers in a scientific way, in which the researchers have limited the results to specific years and fields. Finally, the results were narrowed to 98 research. After a manual scan and careful read, it resulted in banning irrelevant studies and articles. This step narrowed the number of relevant studies to 21 papers that are directly hitting the scope of research.

21 studies were analysed in this research. The date range of these studies was ten years starting from 2010 until 2020 and the results were limited to the field of Business and Management to ignore the studies that are irrelevant to the aim of this study. It was clear that the studies were increasing during the last 5 years. The main focus of the recent studies was related to effective screening, human bias and best candidate fit.

The researchers have chosen websites and journals which had the theme of adapting AI to recruitment and HR. Moreover, articles were traced to the following domains.

As mentioned below in table1, the following websites were selected:

\begin{tabular}{|c|c|c|c|}
\hline \multicolumn{4}{|c|}{ Websites that have been chosen for this study } \\
\hline$\frac{\text { www.recruiter.co.u }}{\underline{\mathrm{k}}}$ & $\frac{\text { www.marketscreener. }}{\underline{\text { com }}}$ & www.hrdailyadvisor.blr.com & www.dzone.com \\
\hline www.hays.co.uk & $\begin{array}{c}\text { www.theglobeandmail. } \\
\underline{\text { com }}\end{array}$ & www.talentlyft.com & $\begin{array}{l}\underline{\text { www.bullhorn.co }} \\
\underline{\mathrm{m}}\end{array}$ \\
\hline www.forbes.com & $\frac{\text { www.hrtechnologist.co }}{\underline{m}}$ & $\underline{w w w . b e n e f i t n e w s . c o m}$ & $\begin{array}{l}\text { www.bloomberg. } \\
\text { com }\end{array}$ \\
\hline
\end{tabular}




\begin{tabular}{|c|c|c|c|}
\hline$\frac{w w w . c h i e f e x e c u t i v e}{\underline{\text { net }}}$ & $\frac{w w w . \text { digitalhrtech.co }}{\mathrm{m}}$ & $\frac{w w w . e c o n o m i c t i m e s . i n d i a t i m e}{\text { s.com }}$ & www.silo.ai \\
\hline \multicolumn{2}{|c|}{ www3.weforum.org } & $\underline{\text { www.gattacaplc.com }}$ \\
\hline
\end{tabular}

Table 1: This table contains the journals that the researchers have chosen.

The researchers have found different articles with different research methods. In other words, it has been found that 07 articles used the Secondary Data method. Moreover, 05 articles used the questionnaire method, and finally, 18 articles used the conceptual method.

\section{AI in HRM}

AI as a term was firstly mentioned by the father of AI, John McCarthy, in 1956. (McCarthy and Minsky, 1950) described $\mathrm{AI}$ as "the science and engineering of making intelligent machines, brilliant computer programs". Problem-solving and one data-driven function control and lead the automation of recruitment by using AI applications in human resources management (HRM) (A, Dimple , Josh Bersin, Gaurav Lahiri, Jeff Schwartz, 2018).

AI seeks to imitate and enhance human intelligence by comparing natural human intelligence to artificial intelligence. $\mathrm{AI}$ as a science model focuses on alleviating and promoting human physical and mental labor through computational intelligent behavioral models, the development of reasoning, learning, computer systems' decision-making, and complex issues that can usually be resolved only by human professionals. The software in today's recruitment markets uses AI-based solutions to help employers scan a considerable number of apps for the best possible candidates. In fact, this is one of the most widely used forms of AI recruitment solutions today. Textkernel and SAP's Resume Matcher are just a few examples of this kind of software. Textkernel can quickly scan thousands of job applications. Resume Matcher compares the applicants with the job description and the Wikipedia job entries, allowing them to rank the applicants according to their job description.

However, the practical use and benefit of AI to support recruitment are contradictory, while on the other hand, it seems that there is a common ground in terms of its potential. For example, if AI follows humanbased decision-making from the data it scans, so if there have been regularities between recruitment and selection, AI will continue to emphasize these features and repeat decisions taken in the past. This is what human decision-making needs to be considered while using AI-based recruitment tools. (Dessler, 2020). To overcome human error, AI is intended to be designed, but the reality is that an algorithm is only as good as the data on which it has been trained. In 2020, technology organizations were likely to be more aware of AI-related bias problems. Therefore, the management of AI bias by inhouse or outsourcing their AI bias problem solving is expected for technology companies. Either way, it is expected that public and government concern about AI bias will grow so that technology businesses will need to adjust their AI strategies to remain competitive and compliant. The issue of AI does not matter how bias will be addressed. Learning from past mistakes, such as Amazon's AI biased recruiting case, is extremely important for organizations. History could learn to learn from. In order to avoid biases and perhaps create even better diversity, create the opportunity to seek an answer.

With the help of AI, it is now more proficient for recruiters to approach and attract talents (Nawaz, 2019a). Recruiters can identify talented candidates by implementing AI in their business strategies. 
Moreover, with the help of AI, recruiters can effortlessly get data on persona and whether it is suitable for the job applied for or not. Monotonous and repetitive tasks will disappear since AI is accountable for dealing with these procedures efficiently. In this way, recruiters can focus more on innovative and strategic issues. AI is intelligently designed to overcome bias during the selection process. Many primary sources of bias like name, age, gender, race, and belief can, unbiasedly, pass through with the support of AI systems.

Moreover, (Erickson, 2018) mentioned that $38 \%$ of groups have already adopted AI in HRM, while the rest assumed to adapt it in the near future. Studies showed that one of the main challenges of adopting AI in organizations HRM is the shortage of skills and the fear of change (Bullhorn, 2018). Based on this methodology's current facts, this study will discuss how the literature has addressed AI's role in the recruitment process in HRM.

\section{Study Motivation and Objectives}

A recent paper, (Nawaz, 2019b) stated that the AI title in the recruitment process lakes literature review studies. This kind of paper also supports and enriches the holistic view of the literature on the topic. This paper's purpose is related to researchers' interest to adapt the technological methods to traditional human resources practices. In particular, the interest has grown because we are experiencing an increasing phase of the exponential growth of technology. Since the word technology stood out from both causes, the topic's technological aspect was chosen for AI. AI plays the lead role because it is one of the issues for trends between different technologies.

This study aims to observe how artificial intelligence is currently used in the recruitment process. The researchers found out that several articles and published papers, especially on the websites, should be gathered and collected to make it easier for readers to find a source that contains many resources and studies related to the use of $\mathrm{AI}$ in the recruitment process. Moreover, it was essential to identify the importance and significance of $\mathrm{AI}$ in the recruitment process.

\section{Literature Review}

Using AI save organizations money and efforts (Vijay Sundaram, 2018); (Jones, 2018), and it could boost the hard and soft skills of recruiters (Luiza Sayfullina, 2018), improving speed and task efficiency (Niehueser and Boak, 2020), as well as building relationships between recruiters and candidates (Othamar Gama Filho, 2018) to result in fining talents unbiasedly (Rebecca Greenfield and Riley Griffin, 2018). Recruiters all over the world have a big challenge to screen the massive number of CV's and applications directly after finalizing the attracting process and jump to start the selection process. (Chris Collins, 2018) reviewed the challenges related to receiving a large number of applicants to be screened and evaluated in which recruiters sometimes find difficult to tackle. He offered AI solutions to serve the processing of these applications via chatbots, in which every single applicant can engage personally with the organization's interactive system. In these interactions, the system can collect information such as, salary expectations, availability, contact information, skills and experiences. One more challenge was 
the talent pool of previous temporary workers. He offered a solution to mobilize and activate larger number of candidates, in which applicants list will connect organizations with new , fresh and up to date candidates. The third challenge was related to the suitable time and place for communication with candidates. The solution that was discussed was adopting AI chatbots, in which this technology will be available all day long in a nonstop action. Three main topics were discussed as categories that will be tackled screening, human bias and best-fit candidate.

\subsection{Screening}

(Forbes, June and 2018, no date; Malini Goyal, 2017) (Malini Goyal, 2017); and (Forbes Coaches Council, 2018) discussed the AI adoption to enhance the screening process of HRM. Natural Language Processing (NLP) is the process in which text is being Transformed into structured and easy to digest data enables a computer to read language effectively. Moreover, Natural Language Generation (NLG) is the reverse NLP transformation, letting the computer write a language, structuring data into text. Both of these technologies have enormous potential in talent acquisition. Therefore, AI could benefit from the behavior and implications of human beings. However, It is a prerequisite for communicating with humans to fully understand human beings' written and verbal communication patterns, which is why $\mathrm{AI}$ is needed to incorporate the processing of natural language successfully. Although (Faliagka and Ramantas, 2012) stated that by analyzing the linguistics used in the text, AI could map the emotional state of a person, there is a chance that If AI failed to be armed up with reliable algorithms to support the understanding of humans verbal and written patterns, the decisions would be untrustworthy.

The digital age has brought enormous benefits to EHRM. But it also got massive amounts of data that are currently primarily handled manually. For example, simple job ads can generate tens of thousands of responses, many of which may be inappropriate, but all of them need to be screened to find targeted talents. In terms of implementing $\mathrm{AI}$ in the recruitment process, the most positive argument is to save money and obtain with almost 100 percent accuracy the real-time result. This can mean that the fastscreening process can benefit both candidates and organizations, allowing HR to understand the candidate better. The AI would already complete the validation and authentication of criteria before the final screening process.

\subsection{Human Bias}

People's bias can influence many aspects like gender, ethnicity, and age. AI can be programmed primarily to ignore a candidate's background. For example, Google began using an internally based recruitment tool called qDroid in 2015, which provides interviewers with more reliable questions based on the position for which the candidate interviews and disregards the applicant's background. Data and predictive analytics are currently being used to predict the likelihood of success for an applicant in the 
role he or she is seeking. Based on job-specific criteria and other criteria linked to an organization's cultural requirements, algorithms calculate each applicant's matching result (Neelie, 2017). Using AI technologies, the screening process can be handled automatically, and it can fight the bias related to human behavior (Cara Heilmann, 2018); (Alexandra Levit, 2017). In other words, AI may be programmed to ignore the background of a candidate. Unconsciously stereotypes sometimes prevent recruiters from seeing candidates' existing skills, and AI can be useful (Alexandra Levit, 2017). Big companies have enrolled AI to fight against bias-related issues while recruiting talents. These companies adopted AI to host digital 'blind auditions,' allowing recruiters to view previous keywords in a resume to evaluate talent better (Savar, 2017). However, AIs are still only instruments, despite their extensive problem-solving skills. If a tool is not calibrated correctly, the desired results are not likely to be produced. This is manifested in the form of AI bias in the context of this discussion. If you feed a biased AI data, then biased results will be generated. (Gold, 2019).

\subsection{Best Fit Candidate}

Once the organizations have a list of potential candidates, they can use automation tools to resume screening, allowing humans to reduce their list. Some tools use keyword analysis to determine the best applicants based on the content of the $\mathrm{CV}$. Other candidates use various tests and questions to find the most promising candidates based on actual performance. The two techniques show more accurate and higher success rates in finding the right person-to-work fit, overall skill-based testing.

This process will involve tackling the source documents' readability scores and other relevant characteristics, then working the best fit into the system. The main reason employees are not successful in their job is that they do not have a cultural fit with their employers. AI has the potential to overcome this problem. Significant companies already use algorithms to match available jobs to the desired openings on a company's job board. In a LinkedIn job posting, applicants' suitability is assessed by trying to dig into their profiles and their job history. LinkedIn does this. Knowing that some job descriptions will be like a model fits, this AI will be more accurate in predicting whether certain participants or candidates within the workplace fit the job description. The human element, however, will remain a vital component of the process. (Alistair Cox, 2018).

During the last few years, the remote working culture has attracted attention, and organizations are always looking to recruit reliable candidates for that objective. Today, Artificial Intelligence tools can help a lot, mainly if you hire remote employees. It is difficult to believe, but AI can help evaluate the candidate's honesty and morality to determine whether they are fit for the job. The company can vet reliable applicants and distribute the workforce accordingly. In fact, for on-demand applications like Uber, Zomato, and so on, this AI functionality has proven to be a huge benefit (Christopher McFadden, 2019). 


\subsection{AI applications}

With the aid of technologies such as the Internet of Things (IoT), big data analysis, cloud computing, and the newly added AI, the digital world of Industry 4.0 has reshaped recruiting and various other HR processes. As an application, AI has been defined as Software and/or hardware systems that can think like human beings and make intelligent decisions based on data (Liu et al., 2018). Organizations are already using multiple AI-enabled applications such as speech and face recognition as well as problemsolving, but HRM is in the initial stages of its implementation. AI completely redefines the employerapplicant relationship. AI tools like Chatbot provide applicants with a new and enhanced employer experience. The candidate assessment process, scheduling the interview, reference checking, and sending job offers to the selected candidates can also be automated by other AI-imbued applications. Only $10 \%$ of companies currently use $\mathrm{AI}$ in a high context, and $36 \%$ of organizations are expected to make full use of $\mathrm{AI}$ in the future (Harver, 2020). Here, the researchers will mention some of the AI applications that are adopted by big companies.

\subsubsection{Fetcher}

www.fetcher.ai

Sony Music and over 500 recruiting teams globally use this AI application within the recruitment process. Fetcher uses the pool of applicant's details, in which it can analyze the data provided and recognize the diversity of each applicant data to suggest the qualified applicants in seconds.

\subsubsection{XOR}

WWW.Xor.ai

McDonald's and IKEA use this AI application. The modern communication trend (Chatbot) is being performed as well as a full application and screening tools like video interviewing and live chats.

\subsubsection{TEXTIO}

\section{www.textio.com}

The researchers mentioned this application earlier in the body of this paper. Moreover, this AI application was also adopted by McDonald's to best deal with human bias-free processes.

\subsubsection{ALLYO}

\section{www.allyo.com}

This application uses interesting user experiences to organizations, in which a full integration can be made with the organization's HR system. It can boost security and support analytical intelligence to show talent acquisition. G4S and the Andersons are one of the companies that adopted ALLYO. 


\subsubsection{Talkpush}

www.talkpush.com

Amazon and Walmart adopted this application to boost communication with applicants to the next stage. In other words, it is a CRM supported communication tool for instant, individualized, real-time conversation using chatbot and video screening techniques.

From the AI as mentioned above applications, it is clear that big companies are using these applications to stop human bias and save time and effort to find qualified talents. However, all of these applications support humans in their task as it is not omitting the human part. In other words, it changes the HRM tasks to become more strategic.

\section{Discussion}

It was clear from the previously mentioned studies that many researchers agree that the use of $\mathrm{AI}$ is beneficial for organizations. To make it more related to our scope, the use of AI in the recruitment process can facilitate dealing with a huge number of applicants, in which screening of resume will take place in a fast and efficient manner (Chris Collins, 2018), (Alistair Cox, 2017), (Cara Heilmann, 2018), (Paul Attfield, 2018), (Ethan Lee, 2018), (Chiradeep BasuMallick, 2018), (Niehueser and Boak, 2020). Other researchers agreed that AI will impact positively on reducing the advantage of knowing a connection or a supporter from within the same organization. At least, if the applicant can't fit in the applied position, corruption in hiring will not be an available option. In other words, the system will definitely not consider any bias attempts (Berta Melder, 2018), (Rebecca Greenfield and Riley Griffin, 2018), (ANZ, 2018), (Savar, 2017), (Alexandra Levit, 2017) (Alistair Cox, 2017).

Also, almost all researchers agreed that AI can boost the process of identifying talents. It is always a challenge for organizations to attract and to hire talented candidates. Hiring talents is one of the most important hiring targets. With the help of $\mathrm{AI}$ and its implementation, it has made it possible for the systems and applications to acquire automation and cognitive insights as well as cognitive engagement in the recruitment process. These technologies would make the system work similar to the human brain in terms of data analysis and the ability to build an effective systematic engagement to process the data in an efficient and fast way. With the development of technologies used in the recruitment process, it has become a target for big organizations to adapt to be competitive in selecting talents from the market pool.

\section{Conclusion}

The research was conducted to make it easier for readers to understand the existing published papers and articles concerning the AI impact on the HR recruitment process. Moreover, this study has summarized the knowledge regarding $\mathrm{AI}$ in the literature. It focuses on the usage and benefits of $\mathrm{AI}$ and its impact on screening, human bias and the best fit candidate recruitment. Moreover, boosting the quality and the time performed during the recruitment processes. Thus, this study will be a good reference to the general topic of the AI recruitment relationship. Recruitment processes along with AI 
benefits were understood in this study such as applicants screening, saving time and efforts, the quality of the output of hiring, and unbiased selections.

AI software was developed to make computers that think logically and behave like humans. HRM has witnessed the efficiency and benefits of AI in the recruitment and hiring processes the ability of AI to adapt to the recruitment has increased rapidly over the last two decades. Recruitment still occurs through traditional methods but is assisted by AI tools and applications. The system helps automate different processes, making decision-making more effective and efficient. The use of AI has improved the hiring process for better quality. Now, HR managers have time to explore HR's bigger picture. Despite advances in technology, however, a major challenge remains in terms of companies' readiness for these new technologies, such as the loss of certain administrative jobs.

This study findings are that the impact of $\mathrm{AI}$ on the recruitment process is beneficial and it improves the practices of the human resources departments. This will also improve the performance and the productivity of any organization as they are selecting the talents from a pool of a huge number of applicants easily. This study has limitations regarding the accessibility of some published papers, but it has covered most of the available papers in this field. The researchers recommend future research to build on the current literature and make a comparison between AI recruitment applications. Also, to Investigate and differentiate the impact of $\mathrm{AI}$ on recruitment in different sectors and geographical locations.

\section{References}

[1] A, Dimple , Josh Bersin, Gaurav Lahiri, Jeff Schwartz, E. V. (2018) A, Dimple , Josh Bersin, Gaurav Lahiri, Jeff Schwartz, E. V. The rise of the social enterprise. Deloitte Insights, 2018. - Google Search. Available https://www.google.com/search?q=A\%2C+Dimple+\%2C+Josh+Bersin\%2C+Gaurav+Lahiri\%2C + Jeff + Schwartz\%2C+E.+V.+The+rise+of+the+social+enterprise.+Deloitte+Insights\%2C+2018.\&o $\mathrm{q}=\mathrm{A} \% 2 \mathrm{C}+$ Dimple $+\% 2 \mathrm{C}+\mathrm{Josh}+$ Bersin $\% 2 \mathrm{C}+$ Gaurav+Lahiri $\% 2 \mathrm{C}+\mathrm{Jeff}+\mathrm{Sch}$ artz $\% 2 \mathrm{C}+\mathrm{E} .+\mathrm{V} .+$ The + ri se+of+the+ (Accessed: 6 September 2020).

[2] Alexandra Levit (2017) Recruitment in the Age of AI and Automation. Available at: https://dzone.com/articles/recruitment-in-the-age-of-ai-and-automation (Accessed: 16 September 2020).

[3] Alistair Cox (2017) AI set to revolutionise recruitment . Available at: https://www.marketscreener.com/quote/stock/HAYS-PLC-4000880/news/Hays-AI-set-torevolutionise-recruitment-25005623/ (Accessed: 17 September 2020).

[4] Alistair Cox (2018) AI will be a big part of our future - but what does that mean for businesses searching for talent? Available at: https://www.hays.co.uk/blog/-/blogs/ai-will-be-a-big-part-ofour-future-but-what-does-that-mean-for-businesses-searching-for-talent- $\quad$ (Accessed: 19 September 2020).

[5] ANZ (2018) Organisations are turning to Artificial Intelligence to improve their recruitment processes. Here's how it can benefit candidates | eFinancialCareers. Available at: https://news.efinancialcareers.com/hk-en/318729/organisations-turning-artificial- 
intelligence-improve-recruitment-processes-heres-can-benefit-candidates-sc (Accessed: 17 September 2020).

[6] Berta Melder (2018) The Role of Artificial Intelligence (AI) in Recruitment. Available at: https://www.talentlyft.com/en/blog/article/207/the-role-of-artificial-intelligence-ai-inrecruitment (Accessed: 19 September 2020).

[7] Bullhorn (2018) UK recruitment trends report. Available at: https://www.bullhorn.com/uk/resources/highlightsfrom-the-2018-uk-recruitment-trendsreport.

[8] Cara Heilmann (2018) Council Post: Artificial Intelligence And Recruiting: A Candidate's Perspective. Available at: https://www.forbes.com/sites/forbescoachescouncil/2018/06/22/artificial-intelligence-andrecruiting-a-candidates-perspective/\#19e600257a88 (Accessed: 16 September 2020).

[9] Chiradeep BasuMallick (2018) 3 Ways Artificial Intelligence (AI) will Transform Recruitment | HR Technologist. Available at: https://www.hrtechnologist.com/articles/recruitmentonboarding/3-ways-ai-will-transform-recruitment/ (Accessed: 17 September 2020).

[10] Chris Collins (2018) 4 ways AI is solving summer hiring challenges | Employee Benefit News. Available at: https://www.benefitnews.com/opinion/4-ways-ai-is-solving-summer-hiringchallenges (Accessed: 16 September 2020).

[11] Christopher McFadden (2019) 7 Amazing Ways Companies Use AI to Recruit Employees. Available at: https://interestingengineering.com/7-amazing-ways-companies-use-ai-to-recruit-employees (Accessed: 17 September 2020).

[12] Dessler, G. (2020) Human Resource Management, 16th Edition. Pearson. Available at: https://www.pearson.com/us/higher-education/program/Dessler-My-Lab-Management-withPearson-e-Text-Access-Card-for-Human-Resource-Management-16thEdition/PGM2569070.html (Accessed: 9 January 2021).

[13] Erickson, R. (2018) Infographic: Insights into a highly mature talent acquisition team.

[14] Ethan Lee (2018) The impact of AI on recruitment | AIHR Digital. Available at: https://www.digitalhrtech.com/impact-ai-recruitment/ (Accessed: 17 September 2020).

[15] Faliagka, E. and Ramantas, K. (2012) 'Application of Machine Learning Algorithms to an online Recruitment System', in ICIW 2012 : The Seventh International Conference on Internet and Web Applications and Services, pp. 215-220. Available at: http://citeseerx.ist.psu.edu/viewdoc/download?doi=10.1.1.885.909\&rep=rep1\&type=pdf (Accessed: 9 January 2021).

[16] Forbes, C. H.-, June, U. and 2018, U. (no date) 'Artificial Intelligence and Recruiting: A Candidate's Perspective'.

[17] Forbes Coaches Council (2018) 10 Ways Artificial Intelligence Will Change Your Career Catherine's Career CornerCatherine's Career Corner. Available at: https://www.forbes.com/sites/forbescoachescouncil/2018/08/10/10-ways-artificialintelligence-will-change-recruitment-practices/\#ccf8bf03a2c5 (Accessed: 17 September 2020). 
[18] Gold, M. (2019) AI for Candidate Screening | Empire Resume, empireresume. Available at: https://empireresume.com/ai-for-candidate-screening/ (Accessed: 9 January 2021).

[19] Harver (2020) 11 Innovative Uses Of AI In Recruitment In 2020 - Harver, Harver. Available at: https://harver.com/blog/ai-in-recruitment-2020/ (Accessed: 11 January 2021).

[20] Jesson, J., Matheson, L. and Lacey, F. (2011) Doing your literature review: Traditional and systematic techniques. Available at: https://books.google.com/books?hl=en\&lr=\&id=LUhdBAAAQBAJ\&oi=fnd\&pg=PP1\&dq=Jesson,+ Matheson,+\%26+Lacey,+2011\&ots=lCdrevKZSh\&sig=Q9ececd7_22WZqQIZoaJ5AOjnPQ (Accessed: 6 September 2020).

[21] Jones, H. (2018) How artificial intelligence can enhance your recruitment process. Available at: https://www.gattacaplc.com/How-artificial-intelligence-can-enhance-your-recruitment-process (Accessed: 19 September 2020).

[22] Leopold, A. T., Ratcheva, V. S. and Zahidi, S. (2018) The Future of Jobs Report 2018 Insight Report Centre for the New Economy and Society. Available at: http://www3.weforum.org/docs/WEF_Future_of_Jobs_2018.pdf (Accessed: 12 September 2020).

[23] Liu, J. et al. (2018) 'Artificial intelligence in the 21st century', IEEE Access, 6, pp. 34403-34421. doi: 10.1109/ACCESS.2018.2819688.

[24] Luiza Sayfullina (2018) Artificial Intelligence and recruiting: Why soft skills matter for an AIrecruiter — Silo.AI. Available at: https://silo.ai/why-soft-skills-matter-for-an-ai-recruiter/ (Accessed: 17 September 2020).

[25] Malini Goyal (2017) 'How artificial intelligence is reshaping recruitment, and what it means for the future of jobs', Bennett, Coleman \& Company Limited, pp. 1-6. Available at: https://economictimes.indiatimes.com/jobs/how-artificial-intelligence-is-reshapingrecruitment-and-what-it-means-for-the-future-of-jobs/articleshow/60985946.cms?from=mdr (Accessed: 16 September 2020).

[26] McCarthy, J. and Minsky, M. (1950) 'Inteligencia Artificial'.

[27] Nawaz, N. (2019a) 'How Far have we come with the study of artificial intelligence for recuritment process', International Journal of Scientific and Technology Research, 8(7), pp. 488-493.

[28] Nawaz, N. (2019b) 'How Far have we come with the study of artificial intelligence for recuritment process', International Journal of Scientific and Technology Research, 8(7), pp. 488-493. Available at:

https://www.academia.edu/download/60659326/How_Far_Have_We_ComeWith_The_Study_Of _Artificial_Intelligence_For_Recruitment-Process20190920-107927-cdbt20.pdf (Accessed: 6 September 2020).

[29] Neelie (2017) 5 Fascinating Uses of AI in Recruitment. Available at: https://harver.com/blog/uses-ai-in-recruitment/ (Accessed: 19 September 2020).

[30] Niehueser, W. and Boak, G. (2020) 'Introducing artificial intelligence into a human resources function', Industrial and Commercial Training, 52(2), pp. 121-130. doi: 10.1108/ICT-10-20190097. 
[31] Othamar Gama Filho (2018) Artificial Intelligence Tech Is Evolving The Recruiting Process. Available at: https://chiefexecutive.net/artificial-intelligence-tech-evolving-recruiting-process/ (Accessed: 17 September 2020).

[32] Paul Attfield (2018) How artificial intelligence is transforming the recruitment process - The Globe and Mail. Available at: https://www.theglobeandmail.com/business/article-how-artificialintelligence-is-transforming-the-recruitment-process/ (Accessed: 16 September 2020).

[33] Rebecca Greenfield and Riley Griffin (2018) Artificial Intelligence Is Coming for Hiring, and It Might Not Be That Bad - Bloomberg. Available at: https://www.bloomberg.com/news/articles/2018-08-08/artificial-intelligence-is-coming-forhiring-and-it-might-not-be-that-bad (Accessed: 17 September 2020).

[34] Savar, A. (2017) 3 Ways That A.I. Is Transforming HR and Recruiting. doi: 10.1080/14746689.2017.1407551.

[35] Vijay Sundaram (2018) From Sourcing to Hire: Artificial Intelligence in Recruiting - HR Daily Advisor. Available at: https://hrdailyadvisor.blr.com/2018/10/02/from-sourcing-to-hireartificial-intelligence-in-recruiting/ (Accessed: 17 September 2020). 